\title{
A Certain Exploration on EEG Signal for the Removal of Artefacts Using Power Spectral Density Analysis through Haar wavelet Transform
}

\author{
B.Paulchamy, \\ Department Of ECE \\ Hindustan Institute of Technology \\ Coimbatore -32 \\ Tamilnadu, INDIA
}

\author{
Ila Vennila, \\ Department of EEE \\ PSG College of Technology \\ Coimbatore -04 \\ Tamilnadu, INDIA
}

\begin{abstract}
Electroencephalogram is the method of recording the electrical activity of the brain via sensors (electrodes) placed on the surface of the scalp. EEGs, which are of the order of microvolt, have greater potential for the diagnosis and treatment of mental and brain diseases and abnormalities. However, EEG signals are subject to various kinds of contaminants (i.e) artifacts which arise from human beings themselves.EEG recordings are often significantly distorted by Eye blinks and eye ball movements which cause changes to the electrical fields around the eye. These interpretations, which are often termed as noises, are however problematic. These are of the order of millivolts. The frequency range of EEG waves is $0-64 \mathrm{~Hz}$ while artifacts occur within the range of $0-16 \mathrm{~Hz}$. Therefore, it becomes a must for the removal of ocular artifacts from the EEG signals. The wavelet based EOG algorithm, when applied to the entire length of the EEG signals, results in thresholding both high frequency and low frequency components, including the non-ocular artifacts zones, but it produces considerable loss in the valuable background EEG activity. The ocular artifacts can be detected by means of visual inspection. But, the EOG correction procedure requires ocular artifacts time zones to be fed as input, which is indeed a tedious process. This necessitates the need for the automatic detection of ocular artifacts zones. In this paper, we have proposed a method for automatically identifying the slow varying artifacts zones, followed by the application of wavelet based adaptive thresholding algorithm to the identified artifacts zones. This preserves the background EEG information. Adaptive thresholding is applied only to the artifacts zones. This method preserves both the low frequency components and the shape of the EEG signal in the non-artifact zones, which is very important in clinical diagnosis. In this paper, we have proposed a thresholding method using Haar Wavelet Transform for the removal of artifacts from the recorded EEG signal.
\end{abstract}

\section{General Terms: Bio Medical Signal Processing}

Keywords: Artifact removal, Electro encephalography, Eye Movement, Wavelet Transform, Adaptive Thresholding.

\section{INTRODUCTION}

The EEG signals are susceptible to various kinds of physiological artifacts such as blinking and movement of eyes, beating of heart and movement of other muscle groups. These artifacts combine with the brain signals, thus making the interpretation of EEG signals complicated. Blinking or movement of eye balls produce large electrical potentials around the eyes called the electrooculogram (EOG). The EOG spreads across the scalp, thus contaminating the EEG signals.
It is often referred as ocular artifacts (OA). Several methods have been proposed for the detection and removal of ocular artifacts. One of the earliest methods used for artifact removal is artifact rejection. However the removal of ocular artifacts by artifact rejection is time consuming and results in the loss of valuable EEG data. The most common method used for the removal of ocular artifacts is the regression in time and frequency domain. Since both these methods share an inherent weakness, these were not found to be much convincing for the removal of ocular artifacts. Nowadays, Independent Component Analysis techniques have been used to correct or remove EEG contaminants. These techniques attempt to "unfix" the EEG signals into some number of underlying components. There are many source separation algorithms, often assuming various behaviours or natures of EEG. Regardless, the principle behind any particular method usually allows "remixing" only those components that would result in "clean" EEG by nullifying the weight of unwanted components. Fully automated artifact rejection methods, which use ICA, have also been developed ${ }^{[1]}$.

This paper shows a new method to remove the ocular artefact from the EEG based on Haar transform and frequency analysis. Transfer of eye-movement activity to EEG can have frequency dependent amplitude and phase characteristics ${ }^{[1]}$. The proposed method is suitable for handling such transfer because the threshold formula is used in the frequency domain. The method is demonstrated with artificial signal-innoise EOG (electro-oculogram) and EEG series. In the EEG noise an event related potential (ERP) is buried as a constant signal and in the EOG noise a changing EOG response (saccadic eye-movement) is simulated before adding the whole series to the EEG series. Also decreasing levels of transfer from EOG on EEG leads are simulated as the EOG artefact diminishes from the frontal to the occipital area. Because of the possible frequency dependent phase characteristics also a time-shift of the EOG is simulated. In this project discusses a method to automatically identify slow varying Artefacts zones and applying wavelet based adaptive thresholding algorithm only to the identified Artefacts zones, which avoids the removal of background EEG information. Adaptive thresholding applied only to the Artefacts zone does not affect the low frequency components in the non- Artefacts zones and also preserves the shape (waveform) of the EEG signal in non-artefact zones which is of very much importance in clinical diagnosis. The subtraction formula, corrects very accurately the influence of the EOG artefact on EEG activity. 


\section{OVERVIEW OF EXISTING ARTEFACTS REMOVAL METHOD}

\subsection{Principal Component Analysis:}

Principal component analysis, which was used by Lagerlundet.al to remove the artefacts from EEG, outperformed all the regression based methods. However, it could not completely separate the ocular artifacts from EEG when both the waveforms had similar voltage magnitudes. The major disadvantages of Principal Component Analysis are that it could not decompose the leads into independent components that are spatially orthogonal and hence cannot be applied for higher order statistical dependencies.

\subsection{Independent Component Analysis:} Independent Component Analysis, which is an extension of principal component analysis, can deal with higher order dependencies also ${ }^{[2]}$. It was developed in the context of blind source separation to obtain approximately independent components ${ }^{[3]}$. But the major disadvantage of Independent Component Analysis is that the Independent Component Analysis lacks the variance maximization property possessed by PCA components.

\section{HAAR WAVELET FOR ANALYZING EEG SIGNAL}

The Haar wavelet's mother wavelet function $\psi(t)$ can be described as

$$
\psi(\mathrm{t})=\left\{\begin{array}{cc}
1 & 0 \leq \mathrm{t}<1 / 2, \\
-1 & 1 / 2 \leq \mathrm{t}<1, \\
1 & \text { otherwise. }
\end{array}\right.
$$

Its scaling function $\phi(t)$ can be described as

$$
\phi(t)= \begin{cases}1 & 1 \leq \mathrm{t}<1 \\ 0 & \text { otherwise }\end{cases}
$$

\subsection{HAAR WAVELET ANALYSIS:}

In our proposed method, we make use of Haar Transform for noise removal. Haar Transform ${ }^{[4]}$, which is the simplest of all the wavelet transforms, cross multiplies a function against the Haar Wavelet with various shifts and stretches. The Haar Transform is much referred as a sampling processs, in which each row of the transformation matrix acts as samples of finer resolution. We make use of Haar Transform because it provides the shortest path $^{[5]}$. Thus, the usage of Haar
Transform decreases the time consumed for the removal of noise.

It is a known fact that the technical disadvantage of Haar Wavelet is that it is not continuous and hence not differentiable. It can be however used as an advantage, because Haar Wavelet affords the signal to be used in sudden transitions, such as monitoring of tool failure in machines ${ }^{[6]}$.

\subsection{The Haar wavelet has several notable properties:}

Any continuous real function can be approximated by linear combinations of $\phi(t), \phi(2 t), \phi(4 t) \ldots . . \phi\left(2^{\mathrm{k}} t\right)$ and their shifted functions. This extends to those function spaces where any function therein can be approximated by continuous functions.

(i) Any continuous real function can be approximated by linear combinations of the constant function, $\psi(t)$ $, \psi(2 t), \psi(4 t) \ldots \ldots \psi\left(2^{\mathrm{k}} t\right) \ldots$ and their shifted QWS functions.

(ii) Orthogonality in the form

${ }_{-\infty}^{\infty} \int^{\infty} 2^{\mathrm{m}} \psi\left(2^{\mathrm{m}} \mathrm{t}-\mathrm{n}\right) \psi\left(2^{\mathrm{ml}} \mathrm{t}-\mathrm{n}_{1}\right) \mathrm{dt}=\partial_{\mathrm{m}, \mathrm{m} 1} \partial_{\mathrm{n}, \mathrm{n} 1}$

Here $\delta_{\mathrm{i}, \mathrm{j}}$ represents the Kronecker delta. The dual function of $\psi(t)$ is $\psi(t)$ itself.

(iii) Wavelet/scaling functions with different scale $m$ have a functional relationship:

$$
\phi(t)=\phi(2 t)+\phi(2 t-1)
$$

\section{IDENTIFICATION OF ARTEFACTS ZONES USING HAAR WAVELET}

Researchers utilized the frequency analysis of EEG signals that are contaminated by EOG signals for the removal of ocular artifacts. They made use of the fact that the spikes that are caused due to the rapid eye blinks and EEG differ in their frequencies and this frequency difference between EEG and EOG signals can be used for the removal of ocular artifacts. However, the correlation between the EEG and EOG signals is futile. Therefore, the detection of ocular artifacts by the singular observation of time domain and frequency domain is inaccurate. Hence, it cannot be used as a proficient method for the detection and removal of ocular artifact.

Our proposed methodology makes use of Haar Wavelet Decomposition for the removal of Ocular Artifacts. The block diagram is shown in figure 1 . 


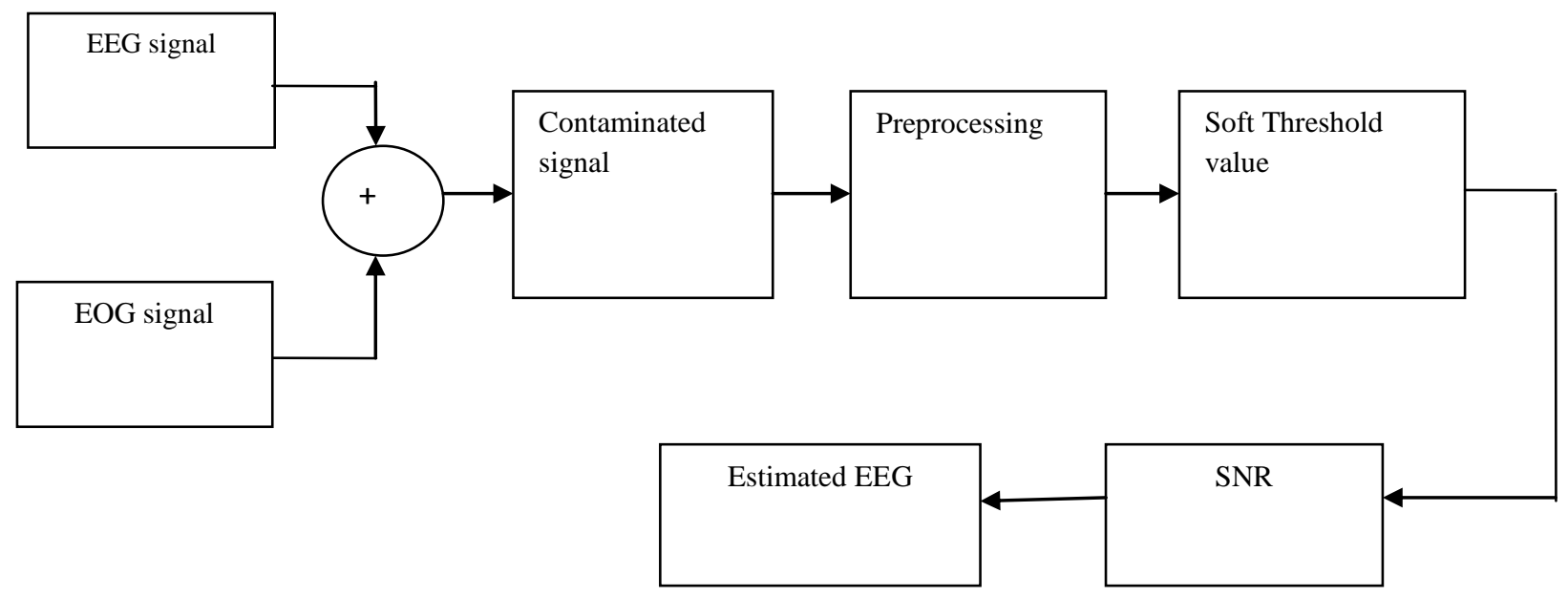

Figure: 1.Block Diagram

In our proposed method, we make use of Haar Wavelet decomposition to denoise the artifacts both effectively and selectively .It is used for the selective detection and removal of artifacts ${ }^{[7]}$. Detection of ocular artifacts by Haar Wavelet decomposition is based on the fact that haar wavelet decomposition can be used to the state when the eye changes from open state to closed state and vice versa.

Using Haar Wavelet Transform, the recorded EEG signal can be decomposed to detect the exact moment when the eye changes from a state of open to closed state and vice versa. When the eye changes its state from open to closed state, decomposition by Haar wavelet transform results in a step function with a falling edge. Similarly, when the eye changes its state from closed to open state, decomposition by Haar Wavelet Transform of the recorded EEG signal results in a step function with rising edge. Our proposed method makes use of the above technique for the removal of artifacts from the contaminated EEG signal. Figure.2.Shows the Identification of Artifacts Zone

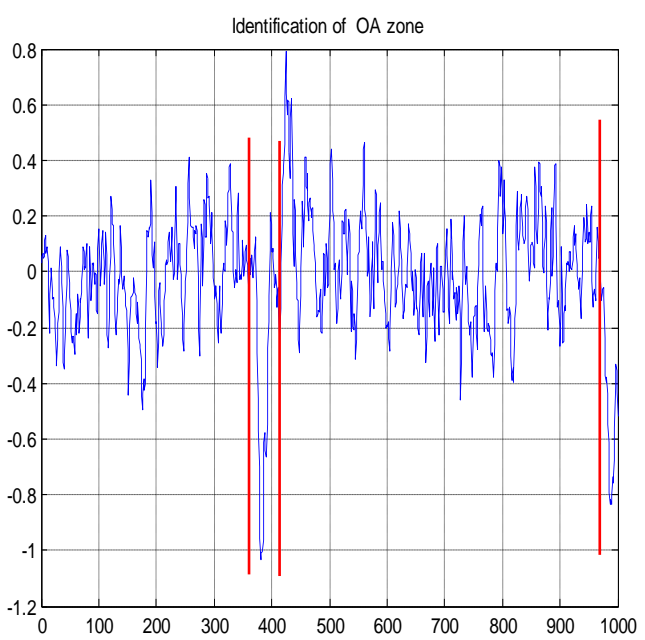

Figure .2 .Identification of artefact zone

\section{ADAPTIVE NOISE CANCELLATION}

Adaptive noise cancellation technique, apart from its application in signal processing and biomedical signal analysis, can also be effectively used to remove noise and artifacts from the EEG signal ${ }^{[8]}$. An effective adaptive noise cancellation requires a reference signal. In our proposed method, a signature of the eye blink signal which is captured from the FP1 and FP2 electrodes (without any artifacts) is used as the reference signal. The input $n(t)$ is the recorded signal which is corrupted by ocular artifacts (EEG+EOG). $r(t)$ is nothing but the output of the non linear channel, which is an estimation of original $\mathrm{EEG}^{[9]}$. This output $\mathrm{r}(\mathrm{t})$,when subtracted from $\mathrm{m}_{\mathrm{rr}}(\mathrm{t})$, produces an error signal $\Psi(\mathrm{t})$, which is nothing but EEG without artifact.The adaptive Noise Cancellation method is Shown in Figure3.

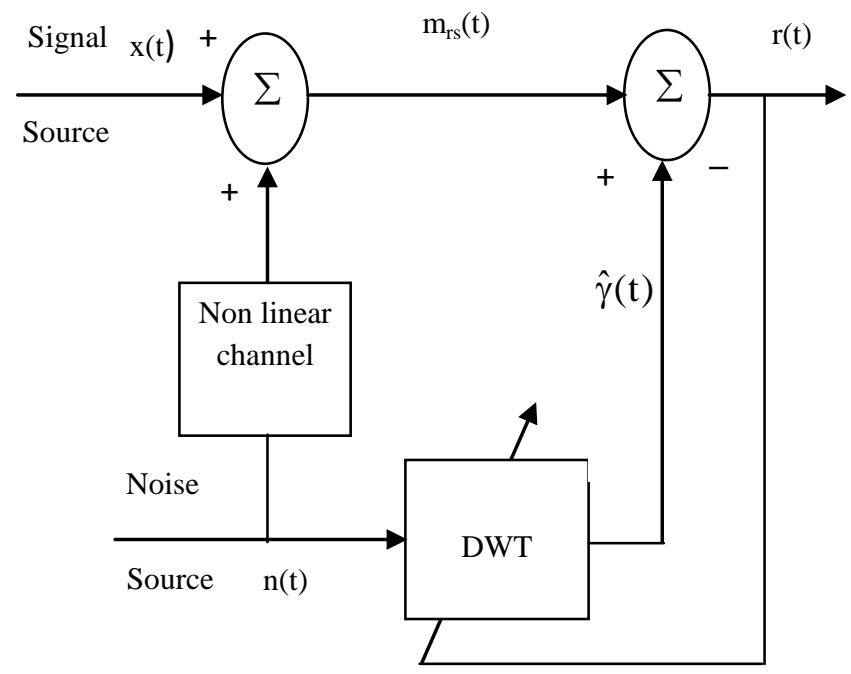

Figure.3.Adaptive noise cancellation 


\subsection{Proposed Methodology}

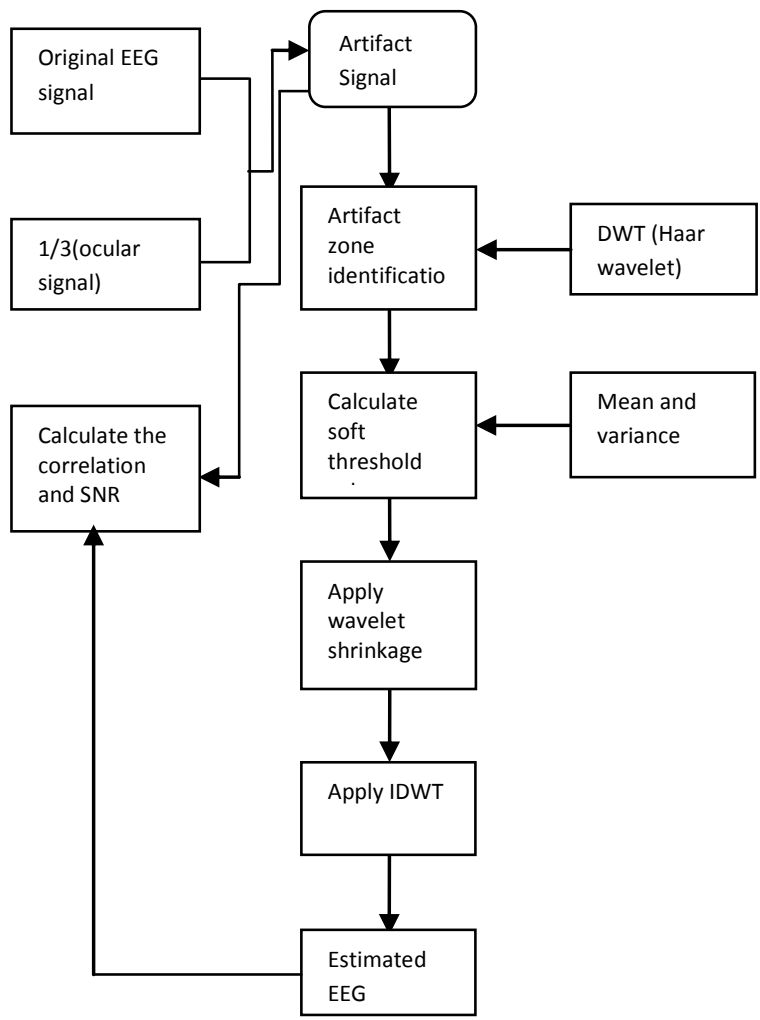

Figure4. Flow chart for the Proposed Method

The flow chart of the proposed method is shown in Figure4.The proposed Algorithm in this paper involves the following steps:

\section{STEP 1:}

Get the original EEG signal and Ocular signal then, combine the one third of Ocular signal with EEG signal to get artifact signal.

\section{STEP 2:}

By applying the Discrete Haar Wavelet Transform the artifacts zones are identified and removed using soft threshold value.

\section{STEP 3:}

The soft threshold value is calculated by usage of mean variance of the signals.

Soft threshold value=1/2(noisy signal+denoisy signal)

In Haar transform itself, it finds the coefficients of the signal. Using that coefficients mean and variance value is estimated and finally finding the soft threshold value.

\section{STEP 4:}

This step involves wavelet shrinkage.

The wavelet shrinkage is a signal denoising technique based in the idea of thresholding the wavelet coefficients. It remove (zero out) the coefficients that are smaller than threshold.

\section{STEP 5:}

Reconstruct the signal using IDWT(inverse discrete Haar wavelet transform)

STEP 6:

Then calculating the PSD and SNR value for different trials and comparing it with original signal.

\section{STEP 7:}

Finally finding the correlation value for output signal and graphically plotting the signal and the justifying the output signal is same as the original signal.

\section{RESULTS\&DISCUSSION}

EEG Data with Artifacts are taken for testing the Proposed Method. The Data is sampled at a rate of 128 Samples per Second. The effect of Artifacts will be dominant in the frontal and front polar channels. Hence it is Sufficient to apply the algorithms to these channels. Figure.4. shows the Original EEG Signal and Figure.5. shows the EOG Corrupted Signal.EEG Signal Mixed with EOG Artifacts which results in the mixing of both EEG and EOG is Called Contaminated Signal ${ }^{[10]}$. The proposed methodology is applied in the Contaminated Signal and the following Results are obtained:

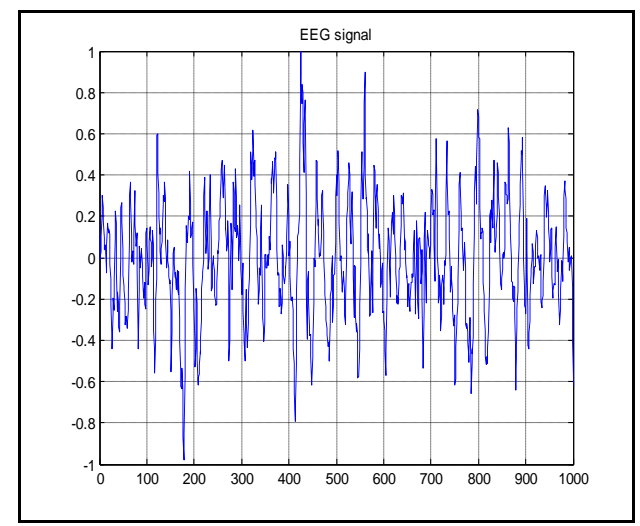

Figure 5.EEG

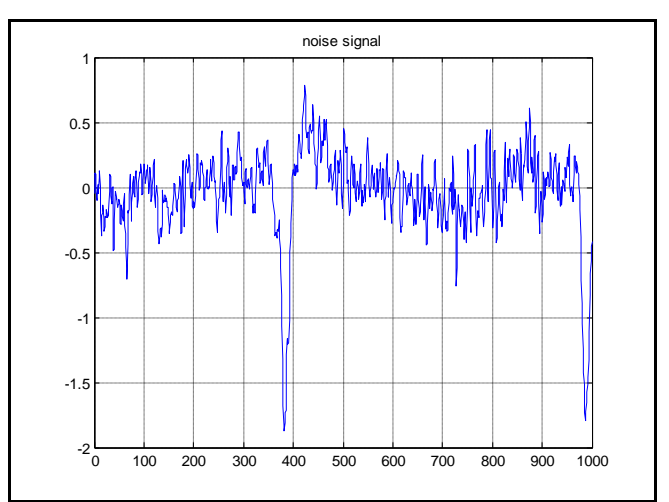

Figure.6.EOG Corrupted Signal

Figure.7 Shows the 10 Second epoch of EOG Contaminated EEG and figure.8. Shows the Corrected EEG Using the proposed Methodology. It clearly shows that the proposed 
Algorithm reduces the amplitude of the Artefacts while preserving the background of EEG.

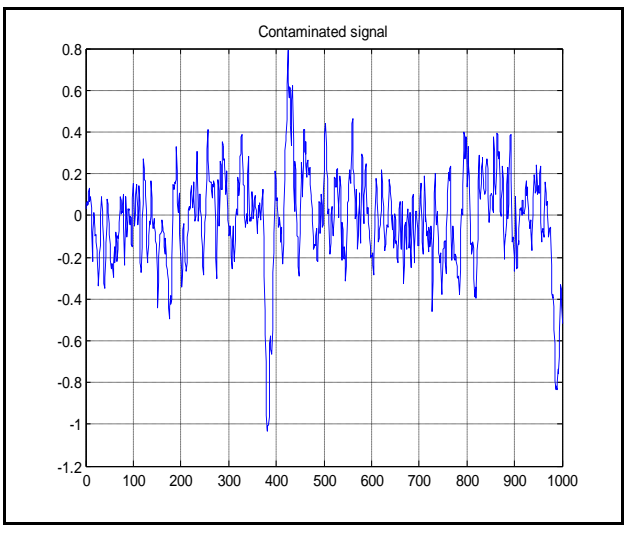

Figure.7.Contaminated Signal

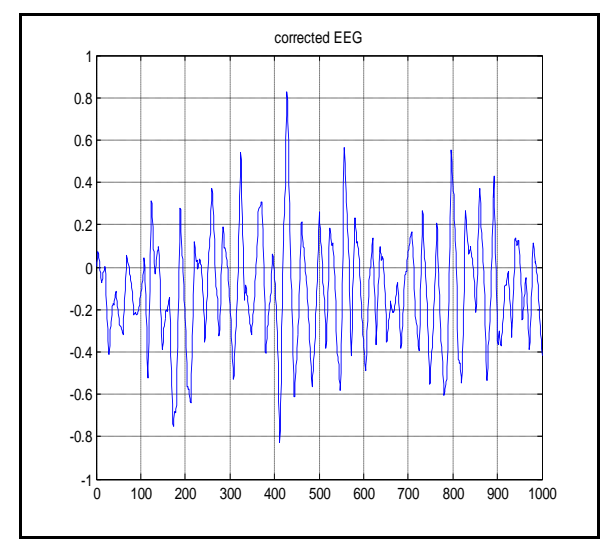

Figure.8.Corrected EEG

Figure.9.Shows the EEG signal corrupted with EOG Artefacts and Denoised EEG Signal from EOG Artefacts

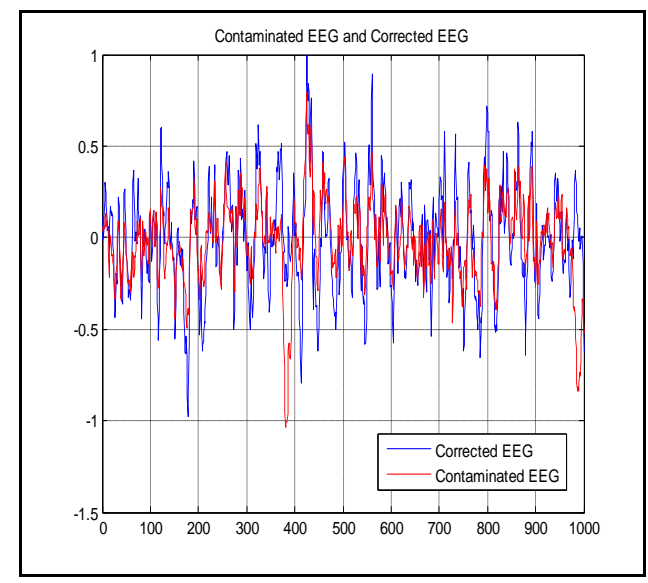

Figure.9.Corrected EEG and Contaminated EEG

Figure.10.Shows the Power Spectra of the Contaminated EEG $(\mathrm{EEG}+\mathrm{EOG})$ and the Corrected EEG. From this figure it is Shown that the powers of the spectral components have been Retanined.

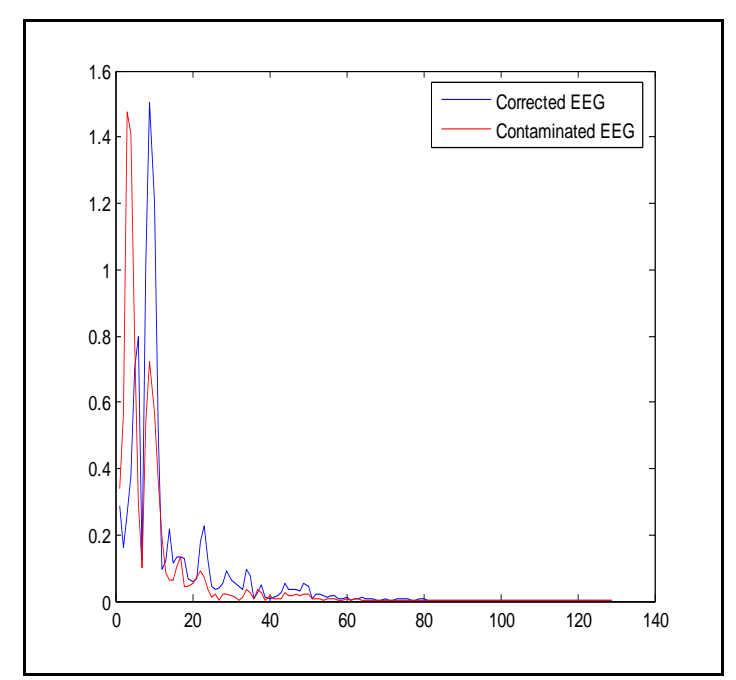

Figure.10.Power Spectral Density plot for Corrected EEG \&Contaminated EEG

The figure.11 shows the correlation plot for the each trail of Noisy EEG and Artefacts Removed EEG.This Shows how close both the signals are in terms of the Shape. (X-Axis Frequency, Y-Axis Correlation Co-efficient)

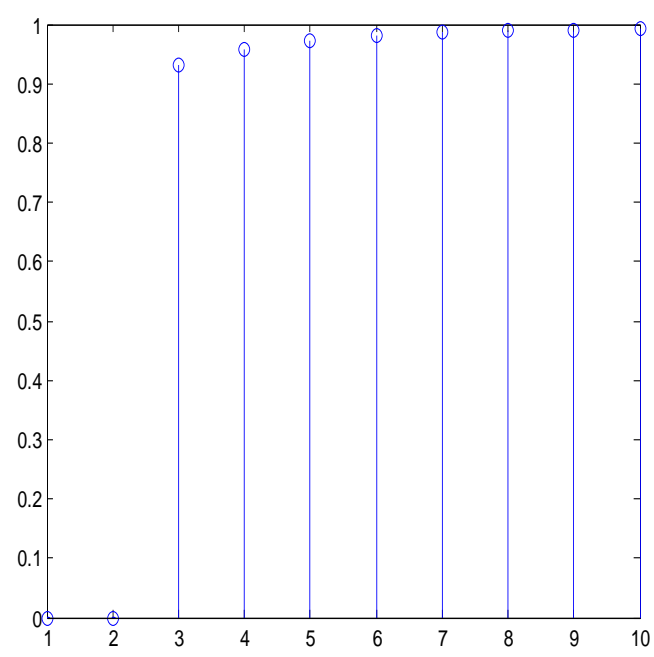

Figure.11.Correlation Plot

Table 1.Summarises the Signal to Noise ratio Comparison of 10 trails of Noisy signal (EEG +EOG Artefact) and Denoised Signal (Corrected EEG). Obviously we can say that the signal to Noise Ratio of denoised signal is higher than the Noisy Signal. 
Table.1.SNR Comparison between Noisy Signal and Denoised Signal

\begin{tabular}{|c|c|c|}
\hline Trails & $\begin{array}{c}\text { SNR for Noised } \\
\text { Signal }\end{array}$ & $\begin{array}{c}\text { SNR for Denoised } \\
\text { Signal }\end{array}$ \\
\hline Trail-1 & 7.9241 & 8.0684 \\
\hline Trail-2 & 10.4229 & 11.5667 \\
\hline Trail-3 & 12.361 & 14.49131 \\
\hline Trail-4 & 13.9447 & 14.061 \\
\hline Trail-5 & 15.2836 & 16.3864 \\
\hline Trail-6 & 16.4435 & 18.5222 \\
\hline Trail-7 & 17.4665 & 20.37981 \\
\hline Trail-8 & 18.3817 & 20.910 \\
\hline Trail-9 & 19.2345 & \\
\hline & & \\
\hline
\end{tabular}

\section{CONCLUSION}

In this paper, a method to identify the artifact through wavelet transform is proposed and soft-like thresholding is applied to the artifact zones. Adaptive thresholding, which is applied only to the artifact zones, does not affect the low frequency components and also preserves the shape of the EEG signal in the non-artifact zones. Power Spectral Density and correlation values are used as performance metrics in this paper. In all cases, Artifacts were adequately attenuated, without removing significant and useful information ${ }^{[11]}$. It is concluded that the proposed method involves less complexity and is a much easier technique for the removal of Artifacts with the help of wavelet decomposition. It is, thus an efficient technique for improving the quality of EEG signals in biomedical analysis

\section{REFERENCES}

[1] R.J. Croft and R.J. Barry, "Removal of artifact from the EEG: a review ", Clinical Neurophysiology, Vol. 30, No.1, (2000), pp. 5 - 19.

[2] P. Levin, E. Urrestarrazu, and J. Gotman, "A system for automatic artifact removal in octal scalp EEG based on independent component analysis and Bayesian classification“, Clinical Neurophysiology, Vol. 117, No.4, (2006), pp. 912- 927.

[3] CA.Joyce, IF.Gorodnitsky, M.Kutas, "Automatic removal of eye movement and blink Artifacts from EEG data using blind component separation ", Psychophysiology. Vol. 41, No.2, (2004),

[4] V. Krishnaveni, S. Jayaraman, et.al, "Automatic identification and removal of Artefacts from EEG Using Wavelet transform ", Measurement Science review, Vol.6, Section 2 No.4.2011.

[5] C. Sidney Burrus, Ramesh A Gopinath and Haitao Guo, "Introduction to Wavelets and Wavelet Transforms ", Prentice-Hall international Inc, (1998).

[6] V. J. Samar, Ajit Bopardikar, Raghuveer Rao, and Kenneth Swartz, "Wavelet analysis of neuroelectric waveforms: A conceptual tutorial ", Brain and Language, 66, (1999), pp. $7-60$.

[7]A.L.Goldberger,AmaralLAN,L.Glass,JM.Hausdorff,Ivanov ich,RG.Mark,JE.Mietus,GB.MOODY,Ck.Peng,HE.Stanl ey,PhysioToolkitand Physionet:Components of a New Reasearch Resource for Complex Physiologic Signals Circulation101(23):e215-e220CirculationElectronicWeb Page httpcic.ahajournals.org/cgi/content/full/101/23/e215:200 0

[8] V. Krishnaveni, S. Jayaraman, N. Malmurugan, A. Kandasamy, D. Ramadoss, "Non adaptive thresholding methods for correcting Artifacts in EEG ", Academic Open Internet Journal, Vol.13, (2004).

[9]Aboitiz, F., Scheibel, A.B., Fisher, R.S., \&Zaidel, E. (1992). Individual differences in brain asymmetries and fiber composition in the human corpus callosum.Brain Research, 598, 154-161.

[10]Barlow, J.S. (1986). Artifact processing (rejection and minimization) in EEG data processing. In F.H. Lopes da Silva, W.S. van Leeuwen, \& A. Remond (Eds.), Handbook of EEG and Clinical Neurophysiological Signals Vol. 2, 15-62.

[11]Barry, R.J. (1976). Failure to find the "local" EEG OR to low- level auditory stimulation. Physiological Psychology, 4,

171-174 